\title{
Energy conserving coupling through small apertures in an infinite perfect conducting screen
}

\author{
J. Petzold, S. Tkachenko, and R. Vick \\ Chair of Electromagnetic Compatibility, Otto-von-Guericke-University, Magdeburg, Germany \\ Correspondence to: J. Petzold (joerg.petzold@ovgu.de)
}

Received: 12 December 2014 - Revised: 14 March 2015 - Accepted: 29 March 2015 - Published: 3 November 2015

\begin{abstract}
Apertures in shielding enclosures are an important issue for determining shielding efficiencies. Various mathematical procedures and theories were employed to describe the coupling between the regions connected via an aperture in a well conducting plane. Bethe's theory describes the coupling via the equivalent problem of field excited dipole moments at the location of the aperture. This approach neglects the reaction of the dipole moments on the exciting field and therefore violates energy conservation. This work emphasizes an analytical approach for coupling between halfspaces through small apertures, inspired by the so called method of small antenna, which allows an understandable generalization of Bethe's theory.
\end{abstract}

\section{Introduction}

In earlier works, Tkachenko et al. (2012) and Rambousky et al. (2013) used the method of small antenna (MSA) to analytically calculate the current induced in small near resonance antennas by external fields in various environments (Tkachenko et al., 2012; Rambousky et al., 2013). The MSA uses the formalism of Green's functions which describe the scatterer and the boundary conditions imposed by the environment. In the vicinity of the scatterer the Green's functions are split in two parts, one singular part and one regular part. The singular part is identified with the singular part of the Green's function for free space, for which asymptotic solutions of the coupling problems are known. The regular part is seen to contain all information of the environment and its integral can be evaluated since the singularity is extracted into the first part. In this way it is possible to construct the induced sources in a specific environment from the source induced in free space and some regularized function representing the specific boundary conditions. This is also called the method of regularization (Nosich, 1999). The scattering of electrically small apertures in the infinite screen can be considered under the approximation of quasi static fields at the aperture, since the fields will not vary much along the aperture. The solution was first published by Bethe (1944), which gives the simple model of magnetic and electric dipole moments, representing the aperture (Bethe, 1944). The assumption of quasi static conditions neglects the influence of radiation of the moments on themselves, which leads to the violation of power conservation (Collin, 1982). So the problem of coupling between two regions separated by an infinite perfect conducting plane via an aperture will be attended by investigating an equivalent problem, which is the induction of electric and magnetic dipole moments located where the aperture is in the original problem. The structure of the present work is to apply the MSA to the dipoles which represent the aperture. This gives an expression for the renormalized moments, which then depend on radiation boundary conditions and fulfill power conservation.

\section{Classical theory}

\subsection{Boundary conditions at the aperture}

At the aperture the fields must fulfill certain boundary conditions. It is useful to split the total fields existing in the considered volume such that

$$
\begin{aligned}
& \boldsymbol{E}=\left\{\begin{array}{lll}
\boldsymbol{E}^{0}+\boldsymbol{E}^{\mathrm{I}} & \text { for } & x<0 \\
\boldsymbol{E}^{\mathrm{II}} & \text { for } & x>0,
\end{array}\right. \\
& \boldsymbol{H}=\left\{\begin{array}{lll}
\boldsymbol{H}^{0}+\boldsymbol{H}^{\mathrm{I}} & \text { for } & x<0 \\
\boldsymbol{H}^{\mathrm{II}} & \text { for } & x>0,
\end{array}\right.
\end{aligned}
$$




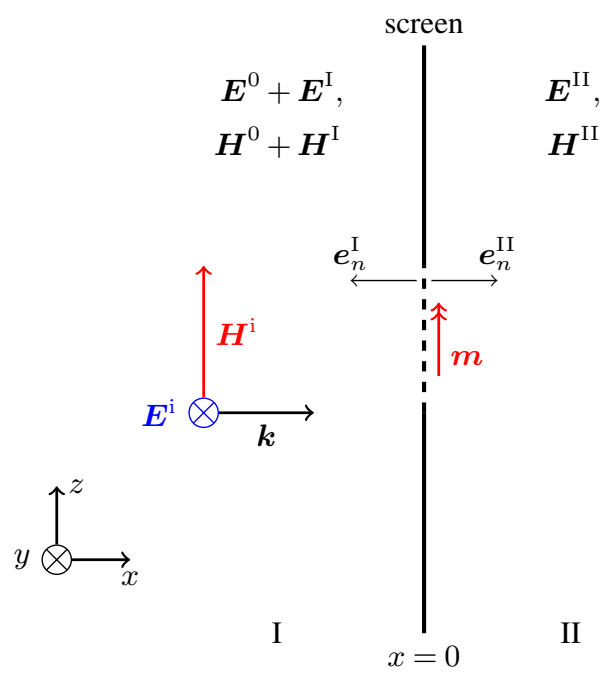

Figure 1. Geometry of the problem with incoming plane wave and induced magnetic moment $m$.

to write the boundary conditions in a convenient way. The fields $\boldsymbol{H}^{0}$ and $\boldsymbol{E}^{0}$ are the shortcut fields, which only exist in the left half space, if the aperture is closed. The other fields are the scattered fields, generated by the presence of the aperture. The tangential electric component and normal magnetic component of the shortcut fields must vanish everywhere at $x=0$. In addition the total fields at the aperture must be continuous. This gives

$$
\begin{aligned}
\boldsymbol{E}_{\perp}^{0}+\boldsymbol{E}_{\perp}^{\mathrm{I}} & =\boldsymbol{E}_{\perp}^{\mathrm{II}}, \\
\boldsymbol{E}_{\|}^{\mathrm{I}} & =\boldsymbol{E}_{\|}^{\mathrm{II}}, \\
\boldsymbol{H}_{\perp}^{\mathrm{I}} & =\boldsymbol{H}_{\perp}^{\mathrm{II}}, \\
\boldsymbol{H}_{\|}^{0}+\boldsymbol{H}_{\|}^{\mathrm{I}} & =\boldsymbol{H}_{\|}^{\mathrm{II}} .
\end{aligned}
$$

The equations Eq. (2) are the boundary conditions at the aperture. Note that they do not depend on size or shape of the aperture (Bethe, 1944).

\subsection{Equivalent sources}

The conditions described by Eq. (2) may be fulfilled by placing equivalent sources at the closed aperture. These sources depend on the geometry of the aperture, the incoming fields and the radiation conditions in both regions and radiate the scattered fields in the respective regions. To find these sources is the first step to solve the coupling problem. To keep the following considerations more simple a polarization of the incident fields is chosen as shown in Fig. 1. Then only Eq. (2d) is needed as it will be illustrated later.

The shortcut fields are the superposition of the incoming and reflected fields in region I only whereas the scattered fields are radiated by the equivalent sources, so with basic electromagnetic theory it can be written for region I

$$
\boldsymbol{H}^{\mathrm{tot}}=\underbrace{\boldsymbol{H}^{\mathrm{i}}+\boldsymbol{H}^{\mathrm{r}}}_{\boldsymbol{H}^{0}}+\underbrace{\operatorname{rot} \boldsymbol{A}_{\mathrm{e}}^{\mathrm{I}}+\frac{1}{j \omega \mu_{0}} \operatorname{grad} \operatorname{div} \boldsymbol{A}_{\mathrm{m}}^{\mathrm{I}}-j \omega \varepsilon_{0} \boldsymbol{A}_{\mathrm{m}}^{\mathrm{I}}}_{\boldsymbol{H}^{\mathrm{I}}},
$$

and for region II

$$
\boldsymbol{H}^{\mathrm{tot}}=\underbrace{\operatorname{rot} \boldsymbol{A}_{\mathrm{e}}^{\mathrm{II}}+\frac{1}{j \omega \mu_{0}} \operatorname{grad} \operatorname{div} \boldsymbol{A}_{\mathrm{m}}^{\mathrm{II}}-j \omega \varepsilon_{0} \boldsymbol{A}_{\mathrm{m}}^{\mathrm{II}}}_{\boldsymbol{H}^{\mathrm{II}}},
$$

with

$$
\begin{gathered}
A_{\mathrm{e}}^{\mathrm{I}}(\boldsymbol{r})=-\boldsymbol{A}_{\mathrm{e}}^{\mathrm{II}}(\boldsymbol{r})=\iint_{S} \overline{\bar{G}}_{\mathrm{h}}^{\boldsymbol{A}_{\mathrm{e}}}\left(\boldsymbol{r}, \boldsymbol{r}^{\prime}\right) \boldsymbol{J}_{\mathrm{e}}^{S}\left(\boldsymbol{r}^{\prime}\right) \mathrm{d} \boldsymbol{r}^{\prime}, \\
\boldsymbol{A}_{\mathrm{m}}^{\mathrm{I}}(\boldsymbol{r})=-\boldsymbol{A}_{\mathrm{m}}^{\mathrm{II}}(\boldsymbol{r})=\iint_{S} \overline{\bar{G}}_{\mathrm{h}}^{\boldsymbol{A}_{\mathrm{m}}}\left(\boldsymbol{r}, \boldsymbol{r}^{\prime}\right) \boldsymbol{J}_{\mathrm{m}}^{S}\left(\boldsymbol{r}^{\prime}\right) \mathrm{d} \boldsymbol{r}^{\prime} .
\end{gathered}
$$

The vector potentials $\boldsymbol{A}_{\mathrm{e}}$ and $\boldsymbol{A}_{\mathrm{m}}$ are associated with the equivalent surface currents $\boldsymbol{J}_{\mathrm{e}}^{S}$ as electric source and $\boldsymbol{J}_{\mathrm{m}}^{S}$ as magnetic source. $S$ is the surface of the aperture. For region II the sign of the vector potentials is negative due to the change of direction of the surface normal vector in the respective region as shown in Fig. $1 . \overline{\bar{G}}_{\mathrm{h}}^{A_{\mathrm{e}}}$ and $\overline{\bar{G}}_{\mathrm{h}}^{A_{\mathrm{m}}}$ are the dyadic Green's functions of the half space which can be found by the mirror procedure and the Green's function of free space. This leads to the conclusion that electric sources do not contribute to the fields, if there is no normal electric field component, because the tangential components of $\overline{\bar{G}}_{\mathrm{h}}^{\boldsymbol{A}_{\mathrm{e}}}$ are zero at the screen. To keep the considerations as simple as possible, a specific configuration as shown in Fig. 1 is chosen. In this configuration only magnetic sources need to be considered.

Interpreting $\overline{\bar{G}}^{\boldsymbol{A}_{\mathrm{m}}}$ as the dyadic vector potential of a dyadic point source, one may write

$\overline{\bar{G}}^{\boldsymbol{H}_{\mathrm{m}}}=\frac{1}{j \omega \mu_{0}}\left(\operatorname{grad} \operatorname{div} \overline{\bar{G}}^{\boldsymbol{A}_{\mathrm{m}}}+k^{2} \overline{\bar{G}}^{\boldsymbol{A}_{\mathrm{m}}}\right)$,

where $\overline{\bar{G}}^{\boldsymbol{H}_{\mathrm{m}}}$ is the dyadic Green's Function for the magnetic field of a magnetic source. Using Eq. (7) on Eq. (3) and inserting in Eq. (2d) the more compact form of an aperture integral equation

$$
-\boldsymbol{H}_{\|}^{0}=2\left(\iint_{S} \overline{\bar{G}}_{\mathrm{h}}^{\boldsymbol{H}_{\mathrm{m}}} \boldsymbol{J}_{\mathrm{m}}^{S} \mathrm{~d} \boldsymbol{r}^{\prime}\right)_{\|}
$$

is achieved.

To evaluate the integrals in Eq. (8) it is necessary to deal with the high order singularities of the Green's functions, which prevent a direct analytical calculation. But by combining the method of analytical regularization and Bethe's theory of diffraction by small apertures an asymptotic analytical solution is possible (Bethe, 1944; Nosich, 1999). 


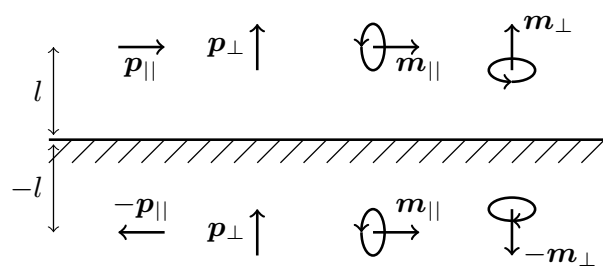

Figure 2. Mirror procedure for electric and magnetic sources.

\section{Generalized theory}

\subsection{Analytical regularization of the Green's function}

The basic idea of analytical regularization is the following. The Green's function representing the interaction between source and field while fulfilling the respective boundary conditions is split into two parts, as

$G=G_{\mathrm{s}}+G_{\mathrm{r}}$.

The singular part $G_{\mathrm{s}}$ represents the interaction of field and source near the scatterer, for which quasi-static solutions exist if the scatterer is electrically small. In case of a small aperture the singular part is Bethe's solution for the quasi-static scattering. The regular part $G_{\mathrm{r}}$ represents the radiation conditions far away from the scatterer, which is in present work the radiation in half space in region I and II. Since the singularity is extracted into the singular part, the integral of the regular Green's function can be evaluated. The problem is now to write $\overline{\bar{G}}_{\mathrm{h}} \boldsymbol{H}_{\mathrm{m}}$ in the form of Eq. (9).

For the half space the Green's function can be found by applying the mirror procedure. The dyadic Green's function for half space is the superposition of the dyadic Green's function for free space of the original and mirrored source.

$\overline{\bar{G}}_{\mathrm{h}}^{\boldsymbol{A}_{\mathrm{m}}}=\frac{1}{4 \pi}\left(\frac{e^{-j k\left|\boldsymbol{r}-\boldsymbol{r}^{\prime}\right|}}{\left|\boldsymbol{r}-\boldsymbol{r}^{\prime}\right|}+\frac{e^{-j k\left|\boldsymbol{r}-\boldsymbol{r}^{\prime \prime}\right|}}{\left|\boldsymbol{r}-\boldsymbol{r}^{\prime \prime}\right|}\right) \overline{\bar{I}}$

The single primed vector $\boldsymbol{r}^{\prime}$ is the position of the original point source, while $\boldsymbol{r}^{\prime \prime}$ is the position of the mirrored point source. In the setup of Fig. 1 this means

$\boldsymbol{r}^{\prime}=\left(x^{\prime}, y^{\prime}, z^{\prime}\right)$

and

$\boldsymbol{r}^{\prime \prime}=\left(-x^{\prime}, y^{\prime}, z^{\prime}\right)$,

which allows together with Fig. 2 to see clearly, that the normal components of $\overline{\bar{G}}_{\mathrm{h}} \boldsymbol{H}_{\mathrm{m}}$ at the screen cancel out each other, while the tangential components at the screen are simply

$\left(\overline{\bar{G}}_{\mathrm{h}}^{A_{\mathrm{m}}}\right)_{\|}=2\left(\overline{\bar{G}}_{\mathrm{f}}^{A_{\mathrm{m}}}\right)_{\|}$

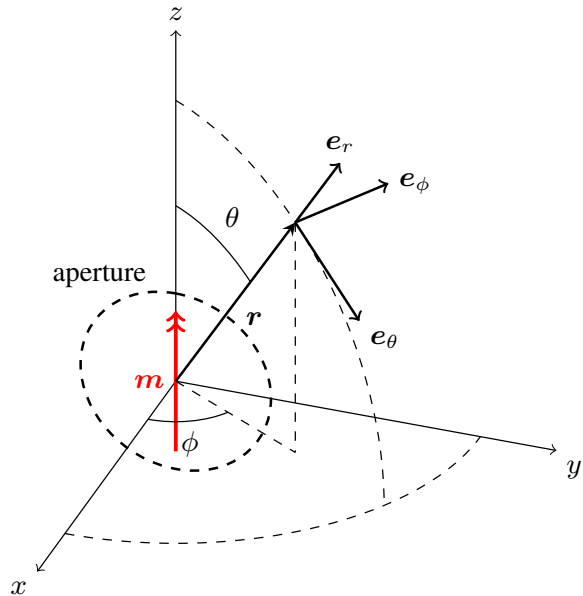

Figure 3. Coordinate systems and aperture.

For the Green's function of free space near a small scatterer regularization can be done by expanding the exponential term into a Taylor series with $k\left|\boldsymbol{r}-\boldsymbol{r}^{\prime}\right| \ll 1$ as the expansion variable around zero, which leads to

$$
\begin{aligned}
\frac{e^{-j k\left|\boldsymbol{r}-\boldsymbol{r}^{\prime}\right|}}{\left|\boldsymbol{r}-\boldsymbol{r}^{\prime}\right|}= & \frac{1}{\left|\boldsymbol{r}-\boldsymbol{r}^{\prime}\right|}-j k-\frac{k^{2}}{2}\left|\boldsymbol{r}-\boldsymbol{r}^{\prime}\right| \\
& +\frac{j k^{3}}{6}\left|\boldsymbol{r}-\boldsymbol{r}^{\prime}\right|^{2}+\ldots
\end{aligned}
$$

By inserting Eq. (12) into Eq. (11) and applying Eq. (7) one arrives at the $z z$-component of the dyadic Green's function for free space as

$$
\left(\overline{\bar{G}}_{\mathrm{f}}^{\boldsymbol{H}_{\mathrm{m}}}\right)_{z z} \approx \frac{1}{4 \pi j \omega \mu_{0}}[\underbrace{\frac{2}{\left|z-z^{\prime}\right|^{3}}+\frac{k^{2}}{\left|z-z^{\prime}\right|}-\frac{2}{3} j k^{3}}_{\text {singular }} \underbrace{}_{\text {regular }}],
$$

which is the only component needed if the incoming magnetic field has only a $z$-component as in the case of the chosen geometry (see Fig. 1). The regular and singular parts can now be clearly distinguished as indicated in Eq. (13).

\subsection{Renormalized Polarizability}

To calculate the induced renormalized magnetic moment Eqs. (13), (11) and (8) are used to derive the expression

$$
-\frac{H_{z}^{\mathrm{i}}}{2}=\iint_{S}\left(\overline{\bar{G}}_{\mathrm{f}, \mathrm{s}}^{\boldsymbol{H}_{\mathrm{m}}}\right) J_{z z} J_{\mathrm{m}, z} \mathrm{~d} \boldsymbol{r}^{\prime}+\left(\overline{\bar{G}}_{\mathrm{f}, \mathrm{r}}^{\boldsymbol{H}_{\mathrm{m}}}\right) \iint_{z z} J_{S}^{S} \mathrm{~m}, z \mathrm{~d} \boldsymbol{r}^{\prime} .
$$

The indices $\mathrm{s}$ and $\mathrm{r}$ relate to the singular and regular parts of the Green's function. On the left hand side of Eq. (14) the boundary condition for the shortcut magnetic field was 


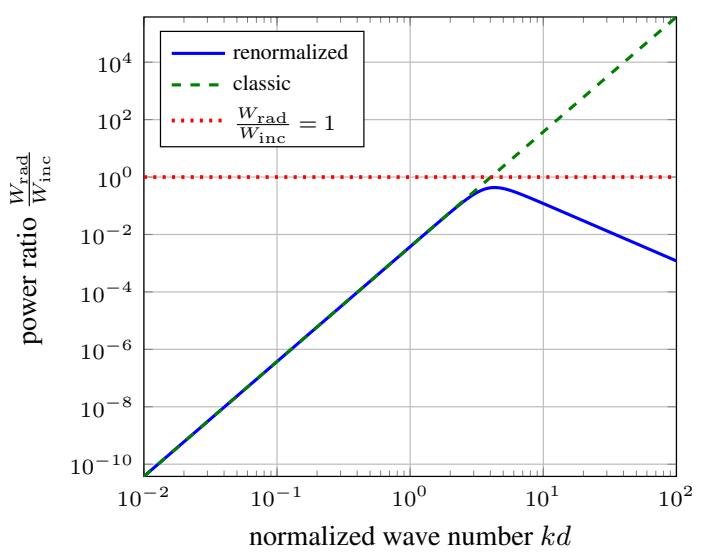

Figure 4. Power ratio over normalized wave number; on logarithmic scale a ratio above 0 , as seen for the classic approach, indicates violation of power conservation.

used. The first integral on the right hand side represents the quasi-static solution by the classical Bethe theory and is the singular part of the magnetic field on the left hand side of Eq. (14). Then the integral can be expressed as

$\iint_{S}\left(\overline{\bar{G}}_{\mathrm{f}, \mathrm{s}} \boldsymbol{H}_{\mathrm{m}}\right)_{z z} J_{\mathrm{m}, z}^{S} \mathrm{~d} \boldsymbol{r}^{\prime}=-\frac{H_{z, \mathrm{~s}}^{\mathrm{i}}}{2}=\frac{m_{z}}{2 \overline{\bar{\alpha}}_{z z}}$

with

$m_{z}=-\overline{\bar{\alpha}}_{z z} H_{z, \mathrm{~s}}^{\mathrm{i}}$

as given by the classical Bethe Theory. The second integral is per definition the magnetic dipole moment

$\iint_{S} J_{\mathrm{m}, z}^{S} \mathrm{~d} \boldsymbol{r}^{\prime}=j \omega \mu_{0} m_{z}$

In Eq. (15) $\overline{\bar{\alpha}}$ is the dyadic magnetic polarizability, which depends on the shape and size of the aperture. For further information on the polarizability see (van Bladel, 2007, p. 489) and (Tesche et al., 1997, p. 210). With Eqs. (15) and (17) inserted in Eq. (14) the magnetic moment may be written as

$$
m_{z}=-\underbrace{\left[\overline{\bar{\alpha}}_{z z}^{-1}+2 j \omega \mu_{0}\left(\overline{\bar{G}}_{\mathrm{f}, \mathrm{r}} \boldsymbol{H}_{z z}\right]^{-1}\right.}_{\tilde{\overline{\bar{\alpha}}}_{z z}} H_{z}^{\mathrm{i}} .
$$

Here $\widetilde{\overline{\bar{\alpha}}}_{z z}$ is the $z z$-component of the renormalized polarizability, which now depends also on the boundary conditions for radiation, which are represented by the first term in the brackets. For other environments such as apertures between cavities and free space one may follow a similar approach. Note that when renormalization is absent Eq. (18) coincides with the classical equation.

\section{Calculation Example}

To demonstrate the improved coupling theory a specific example will be calculated. The focus lies on power calculation and flow. To investigate power conservation, the radiated power of circular aperture is considered. In this case the classical magnetic polarizability is

$\overline{\bar{\alpha}}_{z z}=\frac{d^{3}}{6}$

with $d$ as the diameter of the aperture (Tesche et al., 1997, p. 210). For a circular aperture one gets with Eq. (19) and Eq. (18) for the renormalized polarizability

$\widetilde{\overline{\bar{\alpha}}}_{z z}=\left(\frac{6}{d^{3}}-\frac{j k^{3}}{3 \pi}\right)^{-1}$.

Far from the aperture the scattered fields are the fields radiated by the magnetic dipole moment in half space and with regard to Fig. 3 can be expressed as

$\frac{j k^{2} m_{z}}{2 \pi} e^{-j k r_{0}} \boldsymbol{e}_{\theta} \frac{j \sin \theta}{r_{0}}=\left\{\begin{array}{lll}\boldsymbol{H}^{\mathrm{I}} & \text { if } & 0<\theta \leq \pi \\ \boldsymbol{H}^{\mathrm{II}} & \text { if } & \pi<\theta \leq 2 \pi\end{array}\right.$,

and

$-\frac{j k^{2} m_{z} \eta_{0}}{2 \pi} e^{-j k r_{0}} \boldsymbol{e}_{\phi} \frac{j \sin \theta}{r_{0}}=\left\{\begin{array}{lll}\boldsymbol{E}^{\mathrm{I}} & \text { if } & 0<\theta \leq \pi \\ \boldsymbol{E}^{\mathrm{II}} & \text { if } & \pi<\theta \leq 2 \pi\end{array}\right.$.

Here $\eta_{0}$ is the wave impedance of free space. Note that Eq. (21) are the far fields of a magnetic dipole moment in free space multiplied by 2 since in this case the moment radiates in half space. With Eqs. (21) and (20) the total radiated power in the far field is

$$
\begin{aligned}
W_{\text {rad }} & =\int_{0}^{\pi} \int_{0}^{2 \pi} \boldsymbol{P} r_{0}^{2} \sin \theta \mathrm{d} \theta \mathrm{d} \phi \\
& =\frac{\eta_{0} k^{4}}{3 \pi} m_{z} m_{z}^{*} \\
& =\frac{\eta_{0} k^{4}\left|H_{z}^{\mathrm{i}}\right|^{2}}{12 \pi\left(\frac{9}{d^{6}}+\frac{k^{6}}{36 \pi^{2}}\right)} .
\end{aligned}
$$

If the incoming wave is a plane wave too, the ratio of incoming and radiated power can be calculated as

$$
\begin{aligned}
W_{\text {inc }} & =\eta_{0}\left|H_{z}^{\mathrm{i}}\right|^{2} S, \\
\frac{W_{\text {rad }}}{W_{\text {inc }}} & =\frac{(k d)^{4}}{27 \pi^{2}+(k d)^{6}} .
\end{aligned}
$$

The relation Eq. (24) is plotted in Fig. 4. 

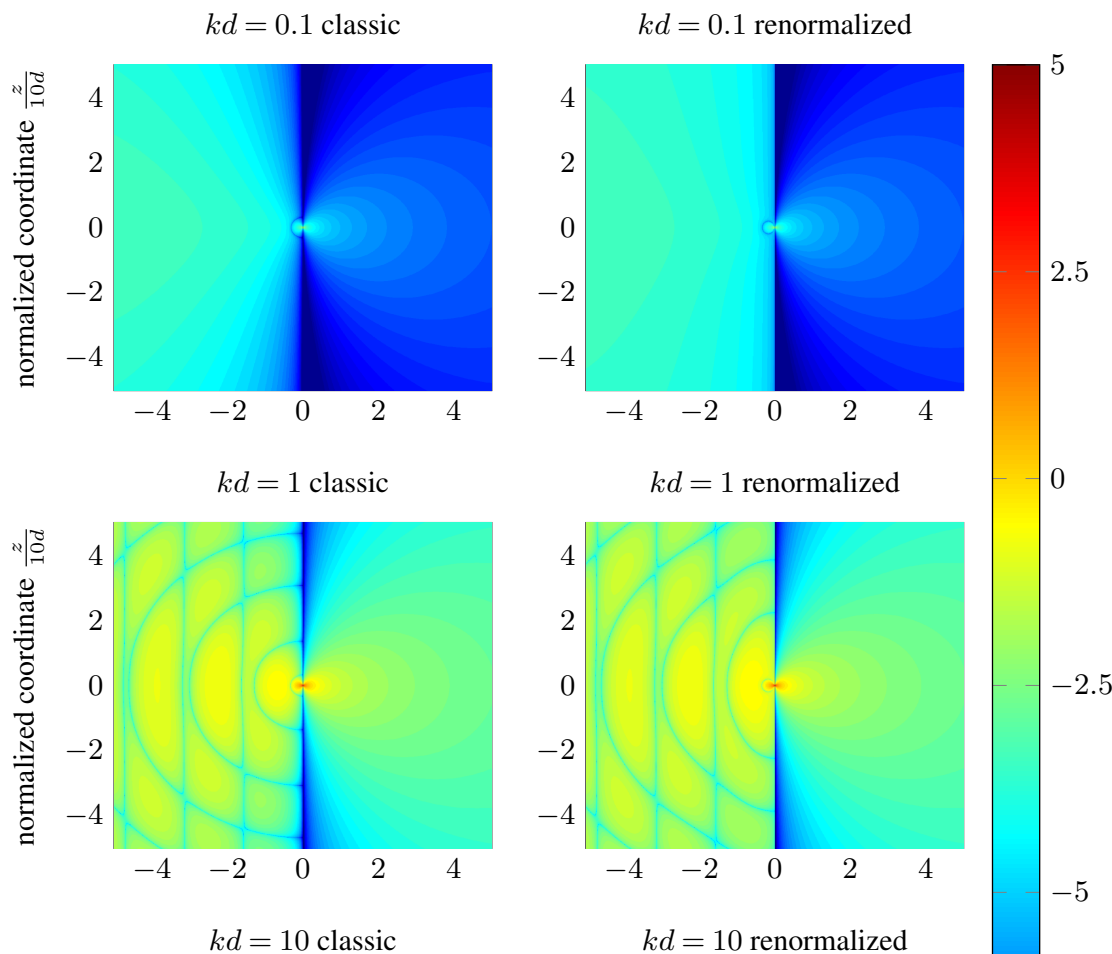

$\vartheta$

$2.5: \Xi$
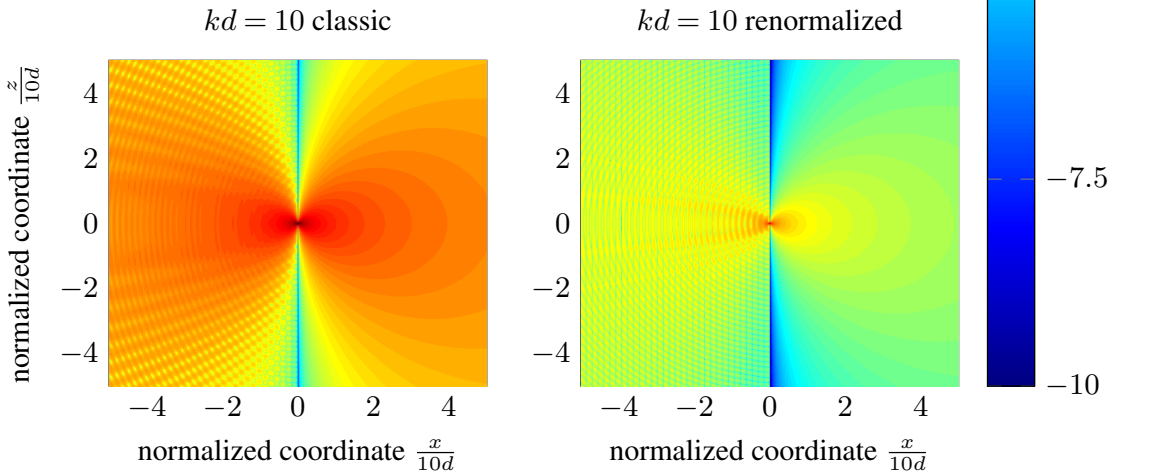

Figure 5. $x$ component of the normalized real power density in the vicinity of the aperture for different normalized wavenumbers. the calculations visualize differences between renormalized and non renormalized moments even for lower frequencies $(k d \leq 1)$.

The difference is also evident in direct analytical calculation of the power density around the aperture. Figure 5 shows the $x$ component of the normalized real part of the total power density calculated with the renormalized and non renormalized aperture moments for different normalized wave numbers. One can see significant change in field distributions even for low frequencies. Note that the calculations for $k d>\frac{\pi}{2}$ are not correct due to neglected multipole moments. One should expect higher angle dependence at this frequencies. This will also be part of future investigations.

\section{Conclusions}

A understandable generalization of a simple model for aperture coupling was derived which features a physical interpretation of aperture radiation. As an example the simplest case of aperture coupling, i.e. an aperture in infinite plane, was used. Calculations of the radiated power of the renormalized moments show that in this way power conservation is established for the model. One has to note, that in the case of radiation in half-space, power conservation is violated for high frequencies, where the dipole model isn't valid at all. But for radiation in cavities and waveguides, the radiation resistance, which is represented by the regular part of the Green's function, can be dominant even for lower frequencies. An application of the presented formalism gives the possibility of forward and backward aperture scattering in different environments. This will be attended in future works.

Edited by: M. Chandra

Reviewed by: two anonymous referees 


\section{References}

Bethe, H.: Theory of Diffraction by Small Holes, Phys. Rev., 66, 163-182, doi:10.1103/PhysRev.66.163, 1944.

Collin, R. E.: Small aperture coupling between dissimilar regions, Electromagnetics, 2, 1-24, 1982.

Nosich, A. I.: The method of analytical regularization in wave-scattering and eigenvalue problems: foundations and review of solutions, IEEE Antenn. Propag. M, 41, 34-49, doi:10.1109/74.775246, 1999.

Rambousky, R., Tkachenko, S., and Nitsch, J.: Calculation of currents induced in a long transmission line placed symmetrically inside a rectangular cavity, in: 2013 IEEE International Symposium on Electromagnetic Compatibility - EMC 2013, Denver, CO, USA, 5-9 August 2013, pp. 796-801, doi:10.1109/ISEMC.2013.6670519, 2013.
Tesche, F. M., Ianoz, M., and Karlsson, T.: EMC analysis methods and computational models, John Wiley \& Sons, New York, USA, 1997.

Tkachenko, S., Nitsch, J., and Al-Hamid, M.: High-Frequency Electromagnetic Field Coupling to Small Antennae in a Rectangular Resonator, Int. J. Antenn. Propag., 1-6, doi:10.1155/2012/897074, 2012.

van Bladel, J.: Electromagnetic fields, 2nd Ed., Wiley-IEEE Press, 1176 pp., 2007. 